\title{
Urology: a long history of innovation
}

\author{
Peter T Scardino
}

At a recent conference, a venture capitalist working in biotechnology announced that his group avoids funding drugs and devices in the field of urology. "Urologists," he said, "are slow to accept new ideas." Having witnessed the rapid adoption of the surgical robot in urology, I was surprised to hear such a comment. On the contrary, I believe that there is a long and distinguished history of innovation in urology.

Although the first endoscopic procedure in medicine was performed on the eye, cystoscopy quickly became the application that established the diagnostic and therapeutic value of endoscopy. The first circulating tumor marker to receive widespread use in clinical practice was acid phosphatase for prostate cancer. Also, the entire field of systemic therapy for cancer was initiated by Charles Huggins' discovery of the response of prostate cancer to castration and estrogens.

In the modern era, extracorporeal shock wave lithotripsy was developed by urologists and rapidly accepted as a treatment for stones, even though it replaced open surgery and competed with the nascent technique of upper urinary tract endoscopy. Expertise in endourology led urologists to perform some of the earliest laparoscopic procedures outside the field of gynecology, including the first pelvic lymph node dissection, nephrectomy and radical prostatectomy. PSA was discovered in the 1970s, and its measurement came into widespread use a decade later; it remains the most utilized circulating marker in oncology. Other advances embraced by urologists have included transrectal ultrasonography coupled with the spring-loaded biopsy gun, prostheses for treating erectile dysfunction and incontinence (i.e. artificial sphincters and slings), and $\alpha$-blockers and 5-a-reductase inhibitors for benign prostatic hyperplasia.

Today, urologists are actively assessing the role of percutaneous ablation of renal masses,
Vast

opportunities

for innovation

exist in the

field of urology,

and safe,

efficacious and

cost-effective

products will

continue to

be readily

embraced

PT Scardino is

Editor-in-Chief

of Nature Clinical

Practice Urology.

\section{Competing interests}

The author declared no

competing interests.

www.nature.com/clinicalpractice doi:10.1038/ncpuro1042 exploring laparoscopic versions of almost all urological procedures, and leading studies of focal ablative therapy for prostate cancer. Several groups have treated incontinence by injecting autologous myoblasts into the urethral sphincter. A urologist helped discover the $V H L$ gene, which led to the development of targeted drugs that are remarkably effective for metastatic renal cell carcinoma. Urologists are the leading users of the surgical robot; in fact, robot-assisted laparoscopic radical prostatectomy essentially saved the surgical robotic industry from an early demise.

Soon robotic surgery will incorporate instruments that allow haptic sensation and improved flexibility, molecular analyses of urine will be used to detect cancer, and gene therapy will be used to treat erectile dysfunction. PET scans will enable visualization of specific molecules, such as the androgen receptor on prostate cancers, and intraoperative probes will use optical or PET technology to help identify cancer at the margins of resection or small metastatic deposits in normal-sized lymph nodes. Antiandrogens that are effective against "hormone-refractory" prostate cancer are also in the therapeutic pipeline.

Still, the marketplace continues to underestimate the possibilities offered by the field of urology and holds back on commercial research and development. In the US, urological conditions, ranging from prostatitis to infertility, account for over 35 million physician visits, 2.5 million emergency room visits and nearly a million hospitalizations each year; annual direct expenditure was well over $\$ 11$ billion in 2000 . As the population ages, the burden of most urological conditions will increase, yet people will expect to be able to extend the length of their lives while maintaining quality of life. Vast opportunities for innovation exist in the field of urology, and safe, efficacious and cost-effective products will continue to be readily embraced. 